\section{Associação de eventos de vida produtores de estresse e mal-estar psicológico: um estudo de base populacional}

\author{
Association of stressful life events and \\ psychological distress: a community-based study
}

Felipe Sparrenberger 1

Iná dos Santos 2

Rosângela da Costa Lima 2

\footnotetext{
1 Departamento de Medicina, Universidade Regional de Blumenau, Blumenau, Brasil.

2 Departamento de Medicina Social, Universidade Federal de Pelotas, Pelotas, Brasil.

Correspondência Felipe Sparrenberger Departamento de Medicina, Universidade Regional de Blumenau.C. P. 1507, Blumenau, SC 89010-971, Brasil. sparrenb@furb.br
}

\begin{abstract}
A cross-sectional study was conducted in 1999 to investigate the association between stressful psychosocial events and psychological distress. A Faces Scale was used to measure the outcome. Death and illness in the family, divorce, robbery, injury, migration, and loss of employment in the previous year were investigated through interviews with 3,942 individuals over 20 years. Logistic regression followed a hierarchical model using socio-demographic characteristics at the first level; psychosocial events at the second; and psychological distress at the third. Injury and migration were not associated with the outcome. The strongest effect was associated with divorce (adjusted OR $=2.34$ ). The highest population-attributable risks were from socioeconomic variables (education $38.2 \%$; family income, $32.3 \%$ ) and the occurrence of at least one event (36.4\%). Public policies aimed at ameliorating adverse social factors such as loss of employment and crime, as well as social support to help individuals manage stressful life events are likely to improve psychological well-being.
\end{abstract}

Life Change Events; Stress; Quality of Life

\section{Introdução}

As evidências mais fortes de que a ocorrência de determinados fatos na vida possa levar a mudanças no estado de saúde provêm do estudo de situações extremas, como participação em combates de guerra 1, envolvimento em acidentes graves 2 ou atos de violência com risco real de perda de vida 3 . Tais situações podem levar a um distúrbio chamado de estado de estresse pós-traumático (DSPT) pela Classificação Estatística Internacional de Doenças e Problemas Relacionados à Saúde - 10a Revisão (CID-10) 4.

A par destes, outros estudos têm buscado associação entre a vivência de fatos não tão dramáticos, os chamados eventos de vida produtores de estresse (EVPE) e a saúde. Os eventos estressantes inerentes à própria vida (ambientais, comunitários, biológicos, abuso ou negligência, traumas e EVPE) poderiam causar desfechos negativos na saúde quando os indivíduos que os experimentam, em função do desgaste físico e psíquico envolvidos, não logram êxito em sua superação, em um determinado tempo 5. O luto normalmente desencadeado pela morte de uma pessoa importante para o indivíduo, por exemplo, de acordo com a CID-10, costuma ser resolvido em um período que pode durar até dois meses.

Alguns autores têm relatado a associação estatística desses eventos com um largo espec- 
tro de agravos à saúde, variando desde esquizofrenia e depressão 6,7, até angina pectoris 8 , cálculos renais sintomáticos 9 , resfriado comum 10 e malformações congênitas 11,12, entre inúmeros outros. Também têm sido realizados estudos visando a verificar não somente a presença de associação, mas também uma possível relação causal entre EVPE e desenvolvimento de sintomas físicos e emocionais 13 .

Os objetivos deste estudo foram investigar o efeito dos EVPE sobre o mal-estar psicológico e o impacto destes eventos sobre a prevalência de mal-estar psicológico na população. A hipótese que motivou este estudo é a de que determinados eventos psicossociais, que apresentem a potencialidade de mudar o curso de uma vida, possam levar a uma ruptura na sensação de sentir-se psicologicamente bem. Esta ruptura será referida como distress ou mal-estar psicológico.

\section{Metodologia}

O delineamento utilizado foi transversal de base populacional. O trabalho de campo foi realizado no último trimestre de 1999, entre a população com mais de vinte anos de idade, residente na zona urbana de Pelotas, município com 330 mil habitantes, localizado no sul do Brasil.

O tamanho de amostra foi calculado para detectar um risco relativo maior ou igual a dois, dada uma prevalência de mal-estar psicológico de $15 \%$ entre os não-expostos. O poder utilizado foi de $80 \%$, erro alfa de $5 \%$ e uma razão de quatro não-expostos para cada exposto. Visando a compensar possíveis perdas ou recusas e para trabalhar fatores de confusão, a amostra foi acrescida respectivamente em $10 \%$ e $20 \%$, atingindo 515 adultos maiores de vinte anos ou 224 domicílios a serem visitados (estimando-se uma média de 2,3 adultos, na faixa etária de interesse, por domicílio). Este número foi acrescido de $50 \%$ prevendo o efeito de delineamento que pode ocorrer na amostragem sistemática e por conglomerado, que foi utilizada 14. Seria necessário, portanto, entrevistar os moradores de 336 domicílios. Uma vez que o estudo foi conduzido sob a forma de consórcio com outros pesquisadores, cujos estudos requeriam uma amostra maior, esta foi expandida para 2.112 domicílios, conferindo ao estudo um poder estatístico de 99,99\%.

Para a seleção da amostra, foi utilizado um processo sistemático por conglomerados. Sortearam-se 48 setores censitários da região urbana do município. Em cada um destes setores foi sorteado um quarteirão, e nele a esquina para iniciar o estudo. O primeiro domicílio considerado foi aquele situado imediatamente à esquerda da casa de esquina (o entrevistador de costas para a casa de esquina). A partir daí, de forma sistemática, foram visitados um em cada três domicílios, sempre no sentido da esquerda para a direita de quem estivesse de costas para a porta do domicílio inicial, até que fosse completado um total de 44 casas. Foram excluídos os visitantes, moradores temporários, empregados domésticos e pessoas com deficiência mental.

Utilizaram-se questionários padronizados e pré-codificados, aplicados por 48 entrevistadoras, todas submetidas a treinamento prévio e participação em estudo piloto. Para o controle de qualidade do trabalho foram vistoriadas pelos supervisores $5 \%$ das residências, selecionadas ao acaso.

\section{Medidas}

\section{- Características sócio-demográficas}

Em cada um dos domicílios selecionados, todos os moradores que preenchessem os critérios de inclusão foram questionados quanto a uma série de características: idade, anos de escola que freqüentou com sucesso e renda familiar no último mês. A cor da pele e o sexo também foram registrados.

\section{- Eventos de vida produtores de estresse}

Pesquisou-se a ocorrência de sete eventos estressantes, aos quais se atribui um valor, geralmente negativo, nos doze meses que antecederam à entrevista. Os eventos foram estudados por intermédio das seguintes questões:

1) Morreu alguém de sua família ou outra pessoa muito importante para o(a) $\operatorname{Sr}(a)$.?

2) $\mathrm{O}$ (a) Sr(a).tem alguma pessoa da família que more na sua casa e que tenha uma doença grave? 3) $\mathrm{O}($ a) $\mathrm{Sr}($ a).perdeu o emprego?

4) $\mathrm{O}(a) \operatorname{Sr}(a)$. se separou do(a) companheiro(a)? 5) $\mathrm{O}($ a) Sr(a). sofreu algum tipo de acidente que precisou de consulta médica?

6) $\mathrm{O}($ a) $\operatorname{Sr}($ a). foi assaltado(a) ou roubado(a)?

7) $\mathrm{O}($ a) Sr(a).é de Pelotas ou veio de outra cidade? E caso tivesse vindo de outra cidade:quando o(a) Sr(a). veio morar em Pelotas?

\section{- Mal-estar psicológico}

Mediu-se a prevalência do desfecho, o mal-estar psicológico, por meio da apresentação da Escala de Faces de Andrews (Figura 1) 15,16, uma 
escala visual intervalar de sete pontos, composta por faces estilizadas, referindo-se ao estado de humor que predominou no ano anterior à entrevista. Cada figura da escala consiste em um círculo, com olhos que não mudam, e uma boca que varia desde um sorriso de quase meio-círculo até um outro meio-círculo, semelhante, voltado para baixo, que inspira o sentimento de tristeza. A entrevistadora formulava a pergunta da seguinte forma: "agora eu vou lhe mostrar uma série de rostos que variam desde uma pessoa que está muito feliz (apontando para a face da escala que mostra alegria máxima) até uma pessoa que está muito triste (apontando para a face correspondente). Qual dessas faces mostra melhor como o Sr(a). se sentiu na maior parte do tempo do último ano?". A resposta foi considerada positiva nos três estados mais negativos (meios-círculos voltados para baixo).

\section{Análise}

$\mathrm{Na}$ análise, foi calculada a prevalência geral de mal-estar psicológico. As associações entre eventos estressantes e mal-estar psicológico foram avaliadas por meio do qui-quadrado de Pearson ou, caso aplicável, teste de tendência linear. Na análise bivariada, calcularam-se as razões de prevalências (RP) brutas e seus respectivos intervalos de confiança de $95 \%$ para testar a força da associação entre o desfecho e as variáveis explicativas.

A análise ajustada do efeito dos eventos estressantes sobre a presença de distress foi feita por regressão logística. Procedeu-se o cálculo das razões de odds ajustadas e do risco atribuível na população (RAP\%) 5 para cada um deles isoladamente e tomando-os em conjunto. Os RAP\% decorrentes das características sócio-demográficas também foram calculados. Para tanto, as variáveis escolaridade e renda foram dicotomizadas, usando-se como ponto de corte, respectivamente, $<12$ e $\geq 12$ anos de educação formal e renda familiar $\leq 6$ e $>6$ salários mínimos (SM) mensais. Foi usada a seguinte fórmula para os cálculos do RAP\%:

$$
\mathrm{RAP} \%=\frac{\mathrm{P}(\mathrm{RO}-1)}{\mathrm{P}(\mathrm{RO}-1)+1}
$$

Onde $\mathrm{P}=$ prevalência do fator e $\mathrm{RO}$ = razão de odds ajustada do fator.

Todas as variáveis foram incluídas na análise multivariada de acordo com um modelo teórico de causalidade (Figura 2). As análises foram realizadas por meio do pacote estatístico Stata 6.0.
Figura 1

Escala de Faces de Andrews.

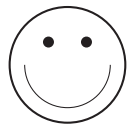

A

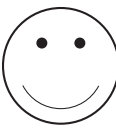

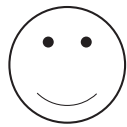

C

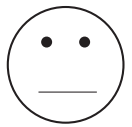

D

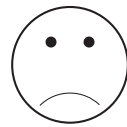

E

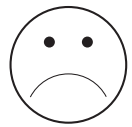

$\mathrm{F}$

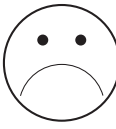

Fonte: McDowell \& Newell 15

Figura 2

Marco teórico.

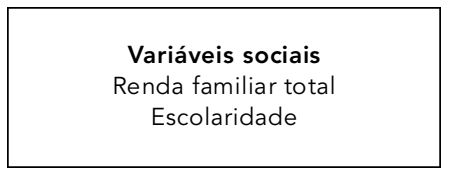

Variáveis demográficas

Sexo

Idade

Cor da pele

\section{Resultados}

Ao todo foram entrevistadas 3.942 pessoas maiores de vinte anos de idade. A prevalência total de distress foi de 14\% (IC95\%: 12,9-15,1).

A Tabela 1 mostra as características sóciodemográficas da amostra. As prevalências de mal-estar psicológico e as RP, com o respectivo intervalo de confiança de $95 \%$, conforme as características sócio-demográficas, são também apresentadas na Tabela 1 . O distress associou-se significativamente com todas as variáveis sócio-demográficas estudadas. As mulheres apresentaram uma prevalência maior do que os homens e, os não-brancos maior do que a dos brancos. Observou-se uma relação direta de 
Prevalência anual de mal-estar psicológico (distress) e medidas de efeito brutas, de acordo com características sociodemográficas. Pelotas, Rio Grande do Sul, Brasil, 1999 ( $n=3.942$ ).

\begin{tabular}{|c|c|c|c|c|}
\hline Características & Freqüência (\%) & Distress (\%) & RP (IC95\%) & p \\
\hline Idade (em anos) & & & & $<0,001^{*}$ \\
\hline $20-29$ & 23,4 & 9,1 & 1,00 & \\
\hline $30-39$ & 21,6 & 10,3 & $1,07(0,81-1,42)$ & \\
\hline $40-49$ & 22,1 & 15,6 & $1,62(1,26-2,09)$ & \\
\hline $50-59$ & 15,2 & 19,3 & $1,98(1,53-2,57)$ & \\
\hline $60+$ & 17,7 & 18,3 & $1,85(1,43-2,39)$ & \\
\hline Cor & & & & $<0,001$ \\
\hline Branco & 80,5 & 12,8 & 1,00 & \\
\hline Não-branco & 19,5 & 19,8 & $1,47(1,23-1,75)$ & \\
\hline Sexo & & & & $<0,001$ \\
\hline Masculino & 42,5 & 9,7 & 1,00 & \\
\hline Feminino & 57,4 & 17,3 & $1,79(1,50-2,13)$ & \\
\hline Renda familiar (em salários mínimos) & & & & $<0,001^{*}$ \\
\hline$<2$ & 20,3 & 21,0 & 1,00 & \\
\hline $2-4$ & 25,4 & 16,8 & $0,81(0,67-0,99)$ & \\
\hline $4,1-6$ & 17,0 & 13,2 & $0,64(0,50-0,81)$ & \\
\hline$>6$ & 37,3 & 9,1 & $0,44(0,36-0,55)$ & \\
\hline Escolaridade (anos completos) & & & & $<0,001$ * \\
\hline 0 & 8,1 & 27,6 & 1,00 & \\
\hline $1-4$ & 21,9 & 19,6 & $0,73(0,58-0,92)$ & \\
\hline $5-8$ & 32,6 & 14,6 & $0,56(0,44-0,7)$ & \\
\hline $9-11$ & 20,3 & 8,2 & $0,31(0,23-0,42)$ & \\
\hline$\geq 12$ & 17,0 & 6,6 & $0,25(0,18-0,35)$ & \\
\hline
\end{tabular}

* Teste qui-quadrado para tendência linear.

mal-estar psicológico com a idade, sendo que pessoas na faixa etária de 50 a 59 anos apresentaram uma prevalência cerca de duas vezes maior do que entre os indivíduos de 20 a 29 anos. Associação inversa foi encontrada com renda e escolaridade, sendo que, quanto maior a escolaridade ou a renda do entrevistado, menor a prevalência observada de mal-estar psicológico. Assim, indivíduos que viviam em famílias com renda mensal total maior do que seis SM apresentaram uma RP cerca de duas vezes menor que a da categoria basal (menor do que dois SM). Em relação à escolaridade, a prevalência de distress foi quatro vezes menor entre indivíduos com mais de doze anos de escolaridade do que entre os analfabetos.

A Tabela 2 mostra uma matriz da distribuição dos eventos estressantes de acordo com as características sócio-demográficas. Morte de pessoa importante e desemprego estiveram associados com as cinco variáveis analisadas. $\mathrm{O}$ fato de ser mulher e de ser menos escolarizado associou-se significativamente à história de apresentar familiar doente (16,3\% entre as mulheres e $13,8 \%$ entre os homens; $15,4 \%$ entre os analfabetos e $11,6 \%$ entre aqueles com doze anos ou mais de escolaridade). Separar-se do cônjuge associou-se com a renda familiar (relação inversa), com a cor (os não-brancos se separando mais do que os brancos) e com a idade (os mais jovens se separando mais do que os mais velhos). A perda do emprego ocorreu para $20,4 \%$ daqueles de menor renda e para $4,8 \%$ daqueles de maior renda ( $\mathrm{p}<0,01$ para tendência linear). Os homens relataram mais desemprego do que as mulheres $(14,7 \%$ e $9,9 \%$, respectivamente). Os não-brancos perderam o emprego na ordem de $18,8 \%$ contra $10,3 \%$ entre os brancos. Os mais jovens perderam mais o emprego do que os mais velhos $(20,9 \%$ na categoria 20 29 anos e $10,2 \%$ na de 50-59 anos). A escolaridade associou-se inversamente com desempre- 
Distribuição dos eventos vitais de acordo com idade, sexo, cor, renda e escolaridade.

Pelotas, Rio Grande do Sul, Brasil, 1999 ( $n=3.942$ ).

\begin{tabular}{|c|c|c|c|c|c|c|c|}
\hline Características & $\begin{array}{c}\text { Morte de } \\
\text { pessoa im- } \\
\text { portante (\%) }\end{array}$ & $\begin{array}{c}\text { Doença } \\
\text { na família } \\
(\%)\end{array}$ & $\begin{array}{c}\text { Separação } \\
\text { conjugal } \\
(\%)\end{array}$ & $\begin{array}{c}\text { Perda do } \\
\text { emprego } \\
\text { (\%) }\end{array}$ & $\begin{array}{c}\text { Roubo/ } \\
\text { Assalto } \\
\text { (\%) }\end{array}$ & $\begin{array}{l}\text { Migração } \\
\text { (\%) }\end{array}$ & $\begin{array}{l}\text { Acidente } \\
(\%)\end{array}$ \\
\hline \multicolumn{8}{|c|}{ Idade (em anos) } \\
\hline $\mathrm{P}$ & $<0,01^{*}$ & $0,48^{*}$ & $<0,01^{*}$ & $<0,01^{*}$ & $0,04^{\star}$ & $<0,01 *$ & $<0,01^{*}$ \\
\hline $20-29$ & 31,2 & 16,5 & 9,7 & 20,9 & 9,1 & 1,8 & 12,0 \\
\hline $30-39$ & 34,5 & 13,7 & 5,7 & 13,3 & 8,1 & 1,4 & 13,4 \\
\hline $40-49$ & 39,0 & 16,9 & 4,5 & 10,4 & 8,1 & 0,1 & 9,5 \\
\hline $50-59$ & 39,0 & 12,9 & 5,4 & 10,2 & 6,2 & 0,2 & 7,9 \\
\hline$\geq 60$ & 43,1 & 15,4 & 1,6 & 1,9 & 6,9 & 0,9 & 8,9 \\
\hline \multicolumn{8}{|l|}{ Cor } \\
\hline $\mathrm{P}$ & 0,001 & 0,067 & $<0,001$ & $<0,001$ & 0,8 & 0,2 & 0,6 \\
\hline Branco & 35,7 & 14,7 & 4,9 & 10,3 & 7,9 & 1,0 & 10,5 \\
\hline Não-branco & 42,0 & 17,4 & 8,5 & 18,8 & 7,7 & 0,5 & 11,1 \\
\hline \multicolumn{8}{|l|}{ Sexo } \\
\hline $\mathrm{P}$ & 0,022 & 0,029 & 0,076 & $<0,001$ & 0,434 & 0,919 & $<0,001$ \\
\hline Masculino & 34,9 & 13,8 & 4,8 & 14,7 & 8,2 & 0,9 & 13,1 \\
\hline Feminino & 38,4 & 16,3 & 6,1 & 9,9 & 7,6 & 0,9 & 8,8 \\
\hline \multicolumn{8}{|c|}{$\begin{array}{l}\text { Renda familiar } \\
\text { (em salários mínimos) }\end{array}$} \\
\hline$P$ & $<0,01^{\star}$ & $0,37 *$ & $<0,01^{\star}$ & $<0,01^{\star}$ & $0,04^{\star}$ & $0,46^{*}$ & $0,23^{*}$ \\
\hline$<2$ & 39,4 & 15,2 & 7,1 & 20,4 & 7,2 & 1,2 & 11,8 \\
\hline $2-4$ & 40,1 & 14,2 & 6,8 & 16,2 & 7,5 & 0,5 & 10,5 \\
\hline $4,1-6$ & 37,2 & 15,5 & 4,6 & 11,7 & 6,1 & 0,6 & 10,2 \\
\hline$>6$ & 33,1 & 16,1 & 4,5 & 4,8 & 9,6 & 1,3 & 10,0 \\
\hline \multicolumn{8}{|c|}{$\begin{array}{l}\text { Escolaridade } \\
\text { (anos completos) }\end{array}$} \\
\hline $\mathrm{P}$ & $<0,01^{*}$ & $0,03^{*}$ & $0,44^{*}$ & $<0,01^{\star}$ & $<0,01^{\star}$ & $<0,01 *$ & 0,97 * \\
\hline 0 & 41,8 & 15,4 & 4,4 & 10,0 & 6,0 & 1,0 & 9,4 \\
\hline $1-4$ & 42,0 & 17,3 & 4,9 & 12,7 & 6,9 & 0,4 & 11,0 \\
\hline $5-8$ & 39,4 & 15,2 & 6,1 & 15,4 & 7,4 & 0,6 & 10,6 \\
\hline $9-11$ & 31,9 & 16,2 & 6,7 & 12,7 & 7,8 & 1,4 & 10,4 \\
\hline$\geq 12$ & 29,6 & 11,6 & 4,9 & 4,3 & 11,0 & 1,8 & 10,5 \\
\hline
\end{tabular}

* teste qui-quadrado para tendência linear.

go. No entanto, aqueles com 5-8 anos de estudo relataram mais desemprego do que os analfabetos $(15,4 \%$ e $10,0 \%$, respectivamente). Ter sido roubado ou assaltado associou-se significativamente e diretamente com renda familiar. $\mathrm{O}$ evento migração mostrou-se associado significativamente apenas com escolaridade e idade, indicando que o perfil daquele que vem residir na cidade de Pelotas é de um indivíduo jovem (o dobro em relação à categoria mais extrema) e com maior escolaridade ( $1 \%$ dos analfabetos contra $1,8 \%$ entre os mais escolarizados). Os homens se acidentaram mais do que as mulhe- res $(13,1 \%$ e $8,8 \%$, respectivamente), e os mais jovens mais do que os mais velhos.

A Tabela 3 mostra a prevalência anual de mal-estar psicológico, bem como as razões de odds bruta e ajustada, de acordo com a vivência de eventos estressantes. Acidente e migração foram os únicos dois eventos que não se associaram significativamente com o desfecho. Os indivíduos que se separaram no último ano, ou que perderam o emprego, tiveram uma RO ajustada mais do que duas vezes maior de referirem distress do que os demais. As ocorrências de doença em familiar e de morte de pessoa 
Prevalência anual de mal-estar psicológico (distress), de acordo com a vivência de eventos estressantes e razão de odds bruta e ajustada. Pelotas, Rio Grande do Sul, 1999 ( $n=3.942)$.

\begin{tabular}{|c|c|c|c|c|}
\hline Evento estressante & Freqüência (\%) & Distress (\%) & RO bruta (IC95\%) & RO ajustada* (IC95\%) \\
\hline \multicolumn{5}{|l|}{ Morte } \\
\hline Não & 63,1 & 12,0 & 1,00 & 1,00 \\
\hline $\operatorname{Sim}$ & 36,9 & 17,6 & $1,57(1,31-1,88)$ & $1,27(1,05-1,55)$ \\
\hline$P$ & & $<0,001(\mathrm{P})$ & $<0,0001(\mathrm{P})$ & 0,0225 (VER) \\
\hline \multicolumn{5}{|l|}{ Acidente } \\
\hline Não & 89,4 & 13,9 & 1,00 & 1,00 \\
\hline Sim & 10,6 & 15,3 & $1,12(0,84-1,49)$ & $1,13(0,83-1,53)$ \\
\hline$P$ & & $0,426(P)$ & $0,4265(P)$ & 0,4776 (VER) \\
\hline \multicolumn{5}{|l|}{ Desemprego } \\
\hline Não & 88,1 & 12,9 & 1,00 & 1,00 \\
\hline Sim & 12,0 & 22,6 & $1,97(1,55-2,51)$ & $1,96(1,49-2,58)$ \\
\hline$P$ & & $<0,001(\mathrm{P})$ & $<0,0001(\mathrm{P})$ & $<0,0001$ (VER) \\
\hline \multicolumn{5}{|l|}{ Separação conjugal } \\
\hline Não & 94,4 & 13,2 & 1,00 & 1,00 \\
\hline Sim & 5,6 & 26,9 & $2,42(1,76-3,31)$ & $2,34(1,66-3,30)$ \\
\hline$P$ & & $<0,001(\mathrm{P})$ & $<0,0001(\mathrm{P})$ & $<0,0001$ (VER) \\
\hline \multicolumn{5}{|l|}{ Roubo } \\
\hline Não & 92,2 & 13,8 & 1,00 & 1,00 \\
\hline Sim & 7,8 & 17,4 & $1,32(0,97-1,80)$ & $1,47(1,05-2,05)$ \\
\hline$P$ & & $0,081(P)$ & $0,0809(P)$ & 0,0333 (VER) \\
\hline \multicolumn{5}{|l|}{ Doença na família } \\
\hline Não & 84,8 & 12,6 & 1,00 & 1,00 \\
\hline Sim & 15,2 & 21,9 & $1,94(1,55-2,42)$ & $1,84(1,45-2,33)$ \\
\hline$P$ & & $<0,001(\mathrm{P})$ & $<0,0001(\mathrm{P})$ & $<0,0001$ (VER) \\
\hline \multicolumn{5}{|l|}{ Migração } \\
\hline Não & 99,1 & 14,0 & 1,00 & 1,00 \\
\hline Sim & 0,9 & 8,3 & $0,56(0,17-1,82)$ & $0,62(0,18-2,15)$ \\
\hline$P$ & & $0,326(P)$ & $0,3258(P)$ & 0,4261 (VER) \\
\hline \multicolumn{5}{|l|}{ Eventos acumulados } \\
\hline Nenhum & 39,2 & 9,3 & 1,00 & 1,00 \\
\hline 1 & 39,0 & 14,1 & $1,62(1,29-2,03)$ & $1,52(1,20-1,92)$ \\
\hline 2 ou mais & 21,7 & 22,1 & $2,78(2,18-3,55)$ & $2,84(2,21-3,64)$ \\
\hline$P$ & & $<0,01(\mathrm{TL})$ & $<0,0001(T L)$ & $<0,0001$ (VER) \\
\hline
\end{tabular}

* Ajustado para escolaridade, renda, sexo e idade.

$\mathrm{P}=\mathrm{p}$ do Qui-quadrado de Pearson; $T L=$ teste de tendência linear; VER = teste de razão de verossimilhança. 
importante para o entrevistado implicaram um aumento, respectivamente, de $84 \%$ e $27 \%$ na RO ajustada, comparativamente aos controles. O maior efeito observado foi para separação conjugal (RO ajustada de 2,34), seguido de desemprego (RO ajustada 1,96) e doença em familiar (RO ajustada de 1,84).

Observou-se um efeito do tipo dose-resposta na avaliação do escore de eventos. Comparativamente aos indivíduos que não apresentaram nenhum evento, os que relataram apenas um tiveram uma RO ajustada de 1,52. Entre os que experimentaram dois ou mais eventos, a RO ajustada foi de 2,84 .

A Tabela 4 mostra o RAP\% dos fatores preditores de distress ajustados para os fatores de confusão. Os mais altos RAP\% foram os das variáveis sócio-econômicas (escolaridade 38,2\% e renda $32,3 \%$ ) e o da ocorrência de pelo menos um dos eventos estressantes $(36,4 \%)$. Se, em todos os grupos, a escolaridade fosse tão alta quanto no grupo de comparação (12 anos ou mais de escolaridade) ou se a renda familiar fosse superior a seis salários mínimos mensais, a prevalência de distress seria reduzida em mais de um terço. Da mesma forma, se os indivíduos entrevistados não tivessem vivenciado nenhum dos EVPE, no ano anterior à entrevista, a prevalência esperada de distress seria $36,4 \%$ menor. Isoladamente, dentre os EVPE estudados, a história de algum familiar morando com o entrevistado e sendo portador de doença grave foi o que apresentou o maior RAP\%, tendo contribuído com $11,3 \%$ da prevalência observada. A separação conjugal, embora apresentando, isoladamente, a associação mais forte com a ocorrência de mal-estar psicológico (RO ajustada de 2,34) contribuiu apenas com $7 \%$ da prevalência observada, uma vez que sua freqüência foi relativamente baixa na população estudada $(5,6 \%)$.

\section{Discussão}

Este estudo teve por objetivo explorar a prevalência e os fatores associados ao mal-estar psicológico. Entende-se bem-estar como o grau em que cada indivíduo julga a qualidade da sua vida favoravelmente como um todo 17. Mal-estar psicológico diferencia-se de depressão, a qual pode ser definida como uma afecção, uma condição clínica suscetível ao diagnóstico por um observador externo, desde que capaz de reconhecer os sinais e sintomas que a definem. O distress é menos específico, resultando da relação entre um evento e o modo como este é interpretado 18 .
Tabela 4

\begin{tabular}{|c|c|}
\hline & $\operatorname{RAP}(\%)$ \\
\hline \multicolumn{2}{|l|}{ Preditor } \\
\hline Renda $<6$ salários mínimos mensais & 32,3 \\
\hline Escolaridade $<12$ anos & 38,2 \\
\hline \multicolumn{2}{|l|}{ Eventos } \\
\hline Morte de pessoa importante & 9,1 \\
\hline Desemprego & 10,3 \\
\hline Separação conjugal & 7,0 \\
\hline Roubo & 3,5 \\
\hline Doença na família & 11,3 \\
\hline 1 ou mais eventos & 36,4 \\
\hline
\end{tabular}

O instrumento de investigação dos EVPE já havia sido testado em estudos anteriores no Brasil 19,20,21. Os resultados mostraram uma confiabilidade de substancial a quase-perfeita (kappa entre 0,6 e 1,0) para a maioria deles 20.

A Escala de Faces utilizada para avaliar a sensação de bem ou mal-estar predominante no último ano, embora ainda não tenha sido validada no Brasil, apresenta um formato nãoverbal, sendo rapidamente assimilada por indivíduos com baixa escolaridade, podendo ser usada, inclusive, por crianças 15,16. É simples de ser aplicada, dispensando a tradução verbal de uma resposta sobre a qualidade de vida do entrevistado. Além disso, mostrou-se um método válido e confiável para diagnosticar bem-estar/mal-estar 15. Também tem sido muito usada em contextos clínicos, inclusive para avaliar a dor 22,23. É ainda necessário, no entanto, que se obtenha evidências sobre sua validade e repetibilidade em estudos conduzidos no Brasil.

Não é possível dizer, com base neste estudo, se os indivíduos que apresentaram mal-estar psicológico receberiam um diagnóstico mais específico (por exemplo, de depressão), caso fosse empregado um outro instrumento ou método diagnóstico. A consistência das associações observadas no atual estudo com as detectadas em outro, realizado na mesma cidade, utilizando o SRQ20 como instrumento diagnóstico 19, no entanto, sugere que ambos estejam medindo dimensões humanas de significado, pelo menos, próximo. Neste citado estudo 19, os autores encontraram uma prevalência aumentada de distúrbios psiquiátricos menores nas mulheres, nos mais idosos, nos mais po- 
bres e menos escolarizados. Além disso, acharam uma associação positiva com todos os eventos estudados, exceto migração e acidente. A definição de distress como sendo as faces da escala que inspiram tristeza (meio-círculo voltado para baixo) sugere um vínculo com o diagnóstico de depressão.

Da mesma forma, o fato de a prevalência de distress haver sido cerca de $40 \%$ menor do que a dos transtornos psiquiátricos menores, naquele citado estudo 19, sugere que a Escala de Faces, tal como foi usada, possa ser um instrumento aplicável para o rastreamento populacional, especificamente, de depressão. A validade da escala como instrumento rastreador de depressão, no entanto, precisa ser testada por meio de estudos especificamente planejados para esse fim.

Os achados deste estudo mostraram que as mulheres, os mais velhos, os não-brancos, os mais pobres e aqueles com menor escolaridade apresentaram maiores prevalências de distress. Entre os sete eventos estressantes estudados, cinco se associaram significativamente com distress: morte de pessoa importante para o entrevistado, desemprego, separação conjugal, roubo e doença na família.

Porém, a significância estatística não diz tudo a respeito do tamanho ou do impacto de uma exposição sobre a saúde. Duas medidas epidemiológicas assumem particular valor na interpretação destes estudos etiológicos: o risco relativo (RR) e a fração etiológica ou risco atribuível na população, em termos percentuais (RAP\%) 1,13,19. Um alto RR tende a aumentar a probabilidade de uma relação causal. Os riscos relativos associados aos eventos não foram particularmente elevados. O RAP\%, definido como o percentual de casos de uma determinada doença ou problema que seriam evitados se a exposição fosse removida, estima o impacto causado pela exposição na comunidade. Os RAP\% associados à renda familiar, à escolaridade e à ocorrência de pelo menos um evento foram bastante elevados.

O uso da amostragem por conglomerados implica a possibilidade de ocorrência de um efeito de delineamento que, quando presente, costuma aumentar a amplitude dos intervalos de confiança. Em que pese o elevado poder estatístico do estudo (99,99\%), não foi levado em conta, na análise, o efeito de delineamento.
Algumas outras limitações poderiam, da mesma forma, ter influído nos resultados observados. Não foram estudados traços de personalidade (definidos em termos da maneira como o sujeito usualmente enfrenta os estressores) 18. Não foi investigada a rede de suporte social, que sabidamente ameniza o impacto dos estressores 18. Também não houve a preocupação em definir características objetivas do evento em si: valor (ganho ou perda, positivo ou negativo), a inevitabilidade, a previsibilidade, a magnitude, a centralidade para o indivíduo ou o impacto físico 24 , nem a percepção subjetiva do mesmo 25 ou tampouco sua duração ou período de extinção 7 . Além disso, só foram estudados eventos negativos, e em pequeno número. As listas de eventos usuais apresentam um elenco de possibilidades bem maior 6,26.

$O$ viés de memória possivelmente tenha provocado apenas pequena alteração nos resultados, uma vez que dados biográficos, diferentemente de dados de personalidade, não são significativamente influenciados por comportamento depressivo ou problemas cognitivos 27. Já o viés de causalidade reversa não pode ser descartado, quando analisada, por exemplo, a relação entre baixa renda e desemprego ou separação conjugal, ou ainda entre esta e o distress.

No plano coletivo, a análise do impacto das variáveis independentes na determinação de distress mostrou que uma melhor distribuição de renda e um maior acesso à educação poderiam reduzir, substancialmente, a prevalência da sensação de não estar se sentindo bem. Ainda, políticas públicas poderiam reduzir a ocorrência de determinados eventos de vida desestruturantes. Isto fica claro quando se pensa na questão do desemprego, da criminalidade e dos indicadores de morbi-mortalidade.

No plano individual, um estudo de intervenção mostrou melhoras objetivas em parâmetros funcionais de asmáticos e de portadores de artrite reumatóide quando esses escreveram a respeito de suas experiências estressantes 28. Embora não tenha sido sugerida a plausibilidade deste achado, entreve-se aí uma nova fronteira na prevenção em termos de Saúde Pública. 


\section{Resumo}

Foi realizado, em 1999, estudo transversal para investigar a associação entre determinados eventos produtores de estresse e a sensação de mal-estar psicológico. A Escala de Faces foi usada para medir o desfecho. A ocorrência de morte e doença em familiar, separação conjugal, roubo, acidente, migração e perda do emprego no ano anterior à entrevista, foi investigada en tre 3.942 pessoas maiores de vinte anos de idade. A análise por regressão logística seguiu modelo hierárquico incluindo, no primeiro nível,características sócio-demográficas individuais; no segundo, eventos estressantes e, no terceiro, o mal-estar psicológico. Acidente e migração não se associaram ao desfecho. $O$ maior efeito foi para separação conjugal (OR ajustada de 2,34). Os mais altos riscos atribuíveis na população foram os das variáveis sócio-econômicas (escolaridade $38,2 \%$ e renda $32,3 \%$ ) e o da ocorrência de pelo menos um dos eventos (36,4\%). Atuação no plano das políticas públicas, visando a reduzir a ocorrência de eventos sociais negativos e, no plano individual, auxiliando as pessoas a lidar melhor com estes fatos da vida, poderiam contribuir para reduzir substancialmente a sensação de mal-estar psicológico.

Acontecimentos que Mudam a Vida; Estresse; Qualidade de Vida

\section{Colaboradores}

F. Sparrenberger participou de todas as fases de produção do artigo, desde o planejamento até a coleta de dados, análise e redação. I. Santos contribuiu no planejamento, na interpretação dos achados e na revisão crítica do manuscrito. R. Lima participou do planejamento e auxiliou na fase de análise e interpretação dos resultados.

\section{Referências}

1. Prigerson HG, Maciejewski PK, Rosenheck RA. Population attributable fraction of psychiatric disorders and behavioral outcomes associated with combat exposure among US men. Am J Public Health 2002; 92:59-63.

2. Koren D, Arnon I, Klein E. Acute stress response and posttraumatic disorder in traffic accident victims: a one-year prospective, follow-up study. Am J Psychiatry 1999; 156:367-73.

3. Brewin CR, Andrews B, Rose S, Kirk M. Acute stress disorder and posttraumatic in victims of violent crime. Am J Psychiatry 1999; 156:360-5.

4. Organização Mundial da Saúde. Classificação estatística internacional de doenças e problemas relacionados à saúde, 10a Revisão. São Paulo: Centro Colaborador da OMS para a Classificação de Doenças em Português; 1995.

5. Jekel JF, Elmore JG, Katz DL. Epidemiologia, bioestatística e medicina preventiva. Porto Alegre: Artes Médicas Sul; 1999.

6. Brown GW, Birley JL. Crisis and life changes and the onset of schizophrenia. J Health Soc Behav 1968; 9:203-14.

7. Livianos-Aldana L, Rojo-Moreno L, Cervera-Martínez G, Domingues-Carabantes JA. Temporal evolution of stress in the year prior to the onset of depressive disorders. J Affect Disord 1999; 53 : 253-62.

8. Billing E, Hjemdahl P, Rehnqvist N. Psychosocial variables in female vs. male patients with stable angina pectoris and matched healthy controls. Eur Hearth J 1997; 18:911-8.

9. Najem RG, Seebode JJ, Samady AJ, Feuerman M, Friedman L. Stressful life events and risk of symptomatic kidney stones. Int J Epidemiol 1997; 26:1017-23.

10. Cobb JMT, Steptoe A. Psychosocial stress and susceptibility to upper respiratory tract illness in an adult population sample. Psychosom Med 1996; 58:404-12.

11. Hansen D, Lou HC, Olsen J. Serious life events and congenital malformations: a national study with complete follow-up. Lancet 2000; 385:875-80.

12. Carmichael SL, Shaw GM. Maternal life stress and congenital anomalies. Epidemiology 2000; 11:30-5.

13. Cooke DJ. The significance of life events as a cause of psychological and physical disorders. In: Cooper B, editor. Psychiatric epidemiology. London: Croom Helm Ltd.; 1987. p. 67-80.

14. Cochran WG. Sampling techniques. New York: John Wiley \& Sons; 1977.

15. McDowell I, Newell C. Measuring health. A guide to rating scales and questionnaires. New York: Oxford University Press; 1996.

16. Lorish CD, Maisiak R. The face scale: a brief, nonverbal method for assessing patient mood. Arthritis Rheum 1986; 29:906-9.

17. Veenhoven R. Questions on happiness; classical topics, modern answers, blindspots. In: Strack F, Argyle M, Schwarz N, editors. Subjective well-being: an interdisciplinary perspective. Oxford: Pergamon Press; 1991. p. 7-26.

18. Palmeira GAES. A medida da psique: uma apreci- 
ação das variáveis utilizadas na investigação dos aspectos psicológicos relacionados ao câncer [Dissertação de Mestrado]. Rio de Janeiro: Escola Nacional de Saúde Pública, Fundação Oswaldo Cruz; 1994.

19. Lima MS, Beria JU, Tomasi E, Conceição AT, Mari JJ. Stressful life events and minor psychiatric disorers: an estimate of the population attributable fraction in Brazilian community-based study. Int J Psychiatry Med 1996; 26: 211-22.

20. Lopes CS, Faerstein E. Confiabilidade do relato de eventos de vida estressantes em um questionário autopreenchido: Estudo Pró-Saúde. Rev Bras Psiquiatr $2001 ; 23: 126-33$.

21. Ustárroz LFL. Eventos estressantes, insatisfação na vida e morbidade psiquiátrica menor em Pelotas, RS [Dissertação de Mestrado]. Pelotas: Universidade Federal de Pelotas; 1997.

22. McKinley S, Coote K, Stein-Parbury J. Development and testing of a Faces Scale for the assessment of anxiety in critically ill patients. J Adv Nurs 2003; 41:73-9.

23. Vihunen R, Sihvonen M. Use of Faces Scale in children's self-assessment of pain. Hoitotiede 1998; 10:123-33.
24. Dohrenwend BP. The role of adversity and stress in psychopathology: some evidence and its implications for theory and research. J Health Soc Behav 2000; 41:1-19.

25. Kuiper NA, Olinger J, Lyons LM. Global perceived stress level as a moderator of the relationship between negative life events and depression. J Human Stress 1986; 12:149-53.

26. Holmes TH, Rahe RH. The social re-adjustment scale. J Psychosom Res 1967; 11:213-8.

27. Bühler KE, Haltenhof H, Geyer S, Bardeleben H. Reliability of biographical data, their relations to personality variables and their influence on lifeevents. J Affect Disord 1999; 53:67-76.

28. Smyth JM, Stone AA, Hurewitz A, Kaell A. Effects of writing about stressful experiences on symptom reduction in patients with asthma or rheumatoid arthritis. A randomized trial. JAMA 1999; 281:1304-9.

Recebido em 11/Fev/2003

Versão final reapresentada em 30/Jun/2003 Aprovado em 23/Out/2003 